\title{
Genome-wide gene expression profiling of ischemia-reperfusion injury in rat kidney, intestine and skeletal muscle implicate a common involvement of MAPK signaling pathway
}

\author{
NAI-JEN CHANG ${ }^{1,2}$, WEN-HUI WENG ${ }^{3}$, KUO-HSUAN CHANG ${ }^{2,4}$, ERIC KAR-WAI LIU ${ }^{1,2}$, CHENG-KENG CHUANG ${ }^{2,5}$, \\ CHIH-CHENG LUO ${ }^{6}$, CHENG-HUNG LIN $^{1,2}$, FU-CHAN WEI ${ }^{1,2}$ and SEE-TONG PANG ${ }^{2,5}$ \\ ${ }^{1}$ Department of Plastic and Reconstructive Surgery, Chang Gung Memorial Hospital, Linkou Medical Center, Tao-Yuan; \\ ${ }^{2}$ College of Medicine, Chang Gung University, Tao-Yuan; ${ }^{3}$ Department of Chemical Engineering and Biotechnology, \\ and Graduate Institute of Biotechnology, National Taipei University of Technology, Taipei; \\ Departments of ${ }^{4}$ Neurology and ${ }^{5}$ Urology, Chang Gung Memorial Hospital, \\ Linkou Medical Center, Tao-Yuan; ${ }^{6}$ Department of Pediatric Surgery, \\ Taipei Medical University Hospital, Taipei, Taiwan, R.O.C.
}

Received November 15, 2013; Accepted June 18, 2014

DOI: $10.3892 / \mathrm{mmr} .2015 .3235$

\begin{abstract}
The mechanisms of ischemia-reperfusion (I/R) injury have not been fully elucidated to date. In order to determine the genetic involvement across different organs during I/R injury, a DNA microarray approach was used to analyze the gene expression profiles of the kidney, intestine, and skeletal muscle in a rat model of I/R injury. Fifteen male Lewis rats were divided randomly into three different organ groups; a sham operation (control group), 60-min-ischemia (Is group) only, and 60-min-ischemia plus 60-min-reperfusion (I/R group), respectively. The target genes were identified by DNA microarray and studied by quantitative polymerase chain reaction (qPCR). By comparing the I/R group with the control group, a 2-fold upregulation of 467, 172, and 3932 and a 2-fold downregulation of 437, 416, and 4203 genes were identified in the kidney, small intestine, and skeletal muscle, respectively. Several commonly upregulated genes associated with mitogen-activated protein kinase (MAPK) pathways, including Jun, Atf3, junB, Fos, Adm and Dusp 1, were differentially expressed in the I/R group. The mRNA expression levels of the target genes were confirmed by qPCR. The present study hypothesized that the MAPK pathway may function in a common pathway of I/R injury and regulate the pathogenesis through activator protein 1 . The findings of the present study
\end{abstract}

Correspondence to: Professor See-Tong Pang, Department of Urology, Chang Gung Memorial Hospital, Linkou Medical Center, 5 Fu-shing Street, 333 Kwei-Shan, Tao-Yuan 33305, Taiwan, R.O.C. E-mail: pst64lab@gmail.com

Key words: ischemia-reperfusion injury, different organs, MAPK pathway, NFאB pathway, activator protein-1 contributed to the understanding of the molecular pathways associated with I/R injury.

\section{Introduction}

Ischemia-reperfusion (I/R) injury was first described in 1968 (1). The influence of $\mathrm{I} / \mathrm{R}$ on various tissues has been widely discussed, since various organs may be affected during traumatic, reconstructive and transplant surgeries $(2,3)$. I/R injury consists of two consecutive components, which comprise ischemia, a breakdown of blood perfusion and reperfusion, where the nutrient blood supply is restored. Ischemia leads to a lack of oxygen within cells of the affected organs, resulting in the conversion of the cellular metabolism to an anaerobic state. This results in lactate accumulation, depletion of cellular adenosine triphosphate, increased production of reactive oxygen species (ROS) and dysfunction of membrane transport systems (4,5). Recent clinical and experimental studies have demonstrated that paradoxically, the major damage of $\mathrm{I} / \mathrm{R}$ injury occurs during the reperfusion period $(6,7)$. Reperfusion initiates complex reactions which lead to the induction of leukocyte accumulation, micro-vascular barrier dysfunction, edema formation, and the release of inflammatory cytokines and complement activation $(8,9)$. The parenchymal damage of I/R injury occurs due to leukocytes being carried to the affected area and the release of inflammatory factors in response to the tissue damage caused by ischemia. The reperfusion reintroduces oxygen that can cause damage to cellular proteins, DNA and the plasma membrane, and results in an increase in release of free radicals, which initiates apoptosis. Leukocytes may additionally act on the capillaries, causing obstruction and leading to increased ischemia $(4,5,10)$.

Numerous organs may be clinically involved in I/R injury. The intestine, kidney and skeletal muscle are the three most affected, in their function, by I/R insult. Studies investigating treatment options for I/R injury are limited in animal 
studies and are rarely conducted in the clinical setting due to the limited understanding of the molecular mechanisms of I/R injury (2). The mechanisms of I/R injury are challenging to study since numerous interacting bioactivities are presented at different time-points. During the shock status, the individual organs suffer from I/R insult, respectively, and receive the toxin from the other organs during reperfusion. Simplification of the method for mechanical study of $I / R$ is important. Tracing the biological changes during I/R at the genomic level is one method that can be employed. There currently are limited reports that have used this approach, and only few pro-inflammatory genes have been identified following I/R insult. These genes include upregulated $S 100 A 4$, complement C4, ADAM2,HO-1, UCP-2 and TMSB4X, and downregulated GLUL, CYP2A6 and $C Y P 2 d 9$ in a renal model; upregulated $M R P 2$ and $P G P$ in an intestinal model, and upregulated $I G F-1$ and $p 27 K p l$ in a skeletal muscle model $(11,12)$. These studies have been limited to individual or small groups of genes, which restrict the exploration of the entire mechanism. There have been no studies, to the best of our knowledge, comparing the genomic changes between different organs under the same I/R insult. In the present study, a kidney, intestine and skeletal muscle model of I/R was used to investigate the genomic changes using a DNA microarray approach, with the aim to identify target genes involved in I/R injury.

\section{Materials and methods}

Animals and experimental groups. A total number of 45 male inbred Lewis rats aged 8-12 weeks with a body weight of 270-330 g were purchased from the National Laboratory Animal Center (Taipei, Taiwan) and used for I/R experiments. All experiments were approved by the Chang Gung Memorial Animal Research and Ethic Committee (Tao-Yuan, Taiwan). During surgery, all animals were placed under a heat lamp to prevent a decrease in body temperature, and during ischemia and reperfusion the exposed organs were covered with normal saline wet gauzes to maintain normal moisture levels. General anesthesia was induced by an intraperitoneal injection of urethane $(15 \mathrm{mg} / \mathrm{kg})$. Rats were assigned to three different study groups, and ischemia and reperfusion injury was studied individually in the kidneys, intestine, and skeletal muscle. Animals in the first group (group I, $n=5$ ) were sham operated and served as controls. Animals in the second group (group II, $\mathrm{n}=5$ ) were subjected to $60 \mathrm{~min}$ of vascular occlusion. Animals in the third group (group III, $\mathrm{n}=5$ ) were subjected to $60 \mathrm{~min}$ of ischemia followed by $60 \mathrm{~min}$ of reperfusion. According to the literature, $60 \mathrm{~min}$ of ischemia and reperfusion were considered appropriate to study early changes in gene expression following I/R injury (2-5,7,23-25).

Establishment of kidney I/R injury. Briefly, the abdomen was opened through a midline incision, and the pedicles of both kidneys were located and freed from surrounding tissue. The left renal artery and vein was clamped with a single microvascular clamp and ischemia was macroscopically verified by a change in color of the kidney to pale blue. For reperfusion studies, the renal clamp was removed and reestablishment of blood flow was again monitored macroscopically.
Establishment of intestinal I/R injury. To study the effects of experimental ischemia on gene expression within intestinal tissue, rats were laparotomized through a midline incision. Briefly, the superior mesenteric artery (SMA) and the supplied intestine were identified and the superior mesenteric vessels were freed from the surrounding tissue. The SMA and superior mesenteric vein (SMV) were occluded with a single vascular clamp for $60 \mathrm{~min}$ and ischemia was verified macroscopically by observing the color change of the intestinal segment to a dark pale color. For reperfusion, the clamp of the superior mesenteric vessels was removed and biopsies were taken after $60 \mathrm{~min}$.

Establishment of skeletal muscle I/R injury. The rat hind-limb vascular occlusion model was used to study the impact of ischemia and reperfusion in the skeletal muscle. Briefly, an incision in the inner side of the hind leg, from the inguinal ligament to the tendon calcaneus insertion, was made. Other than the femoral vessels, all of the muscles, tendons, nerves and vessels were dissected and the femur head was dislocated from the acetabulum. Next, the femoral artery and vein were clamped with a single vascular clamp. For reperfusion, the clamp occluding the femoral vessel was removed to regain of the blood supply to the distal limb was monitored macroscopically.

Organ tissue collection and RNA preparation. At the endpoint of the study, organs subjected to ischemia and reperfusion were harvested under terminal anesthesia. The organs were carefully removed, gently rolled on cotton swabs and irrigated with normal saline to remove the adjacent tissue and excess blood. The organs were then blotted dry, weighed and shock-frozen in liquid nitrogen for storage and subsequent RNA extraction.

The tissue was homogenized and total RNA isolated using TRIzol ${ }^{\mathrm{TM}}$ reagent (Gibco-BRL, Carlsbad, CA, USA) according to the manufacturer's instructions. Subsequently, two phenol/chloroform extractions were performed, followed by a DNAse digestion. Total RNA from the organs of individual rats of each experimental group was pooled and poly $\mathrm{A}^{+}$ RNA (mRNA) isolated with oligo (dT) cellulose columns (Gibco-BRL). Both total RNA and poly $\mathrm{A}^{+}$RNA concentrations were determined spectrophotometrically at $\mathrm{A}_{260}$ and all samples were checked by formaldehyde gel electrophoresis.

Microarray experiment. The samples were prepared for microarray analysis according to the Nimblegen gene expression analysis protocol (Roche Diagnostics, Manheim, Germany). Double-stranded (ds) cDNA from $10 \mu \mathrm{g}$ of total RNA was synthesized using the SuperScript ${ }^{\mathrm{TM}}$ Double-Stranded cDNA Synthesis kit (Invitrogen Life Technologies, Carlsbad, CA, USA). The cDNA was treated with RNase and the total RNA was purified using phenol/chloroform/isoamyl alcohol $(25: 24: 1 \mathrm{v} / \mathrm{v})$ and precipitated by adding $16 \mu \mathrm{l}$ of $7.5 \mathrm{M}$ ammonium acetate, $7 \mu \mathrm{l}$ glycogen ( $5 \mathrm{mg} / \mathrm{ml}$ stock solution), $326 \mu \mathrm{l}$ ice-cold absolute ethanol. The resulting pellet was washed with $500 \mu \mathrm{l}$ ethanol (80\%) and dissolved in $20 \mu \mathrm{l}$ water. Gel electrophoresis was used to verify successful dscDNA synthesis, which was confirmed by the presence of a smeared band of 500-2,000 bp. The reactions were labeled with Cy3-9mer primers using a Nimblegen One-Cola DNA Labeling kit, followed by precipitation using $\mathrm{NaCl}$ and isopropanol. The precipitate was resuspended in $25 \mu \mathrm{l}$ distilled water. 
Table I. Total number of up- and downregulated genes in the kidney, intestine and skeletal muscle.

\begin{tabular}{|c|c|c|c|c|c|c|}
\hline \multirow[b]{2}{*}{ No. of genes } & \multicolumn{2}{|c|}{ Kidney } & \multicolumn{2}{|c|}{ Intestine } & \multicolumn{2}{|c|}{ Skeletal muscle } \\
\hline & Is & $\mathrm{I} / \mathrm{R}$ & Is & $\mathrm{I} / \mathrm{R}$ & Is & $\mathrm{I} / \mathrm{R}$ \\
\hline Upregulated genes & 903 & 467 & 76 & 172 & 2658 & 3932 \\
\hline Downregulated genes & 1351 & 437 & 429 & 416 & 1972 & 4203 \\
\hline
\end{tabular}

Is, ischemia group (group II); I/R, ischemia/reperfusion group (group III).

Microarray hybridization and data analysis. Microarray hybridization was combined with $4 \mu \mathrm{g}$ cDNA from each of the samples. A NimbleGen Hybridization kit (NimbleGen Systems; Roche) was used for the hybridization reaction according to the manufacturer's instructions. The hybridization reaction was performed in a MAUI Hybridization system (BioMicro ${ }^{\circledR}$ Systems, Inc., Salt Lake City, UT, USA). Following hybridization, the array was washed and dried according to the NimbleGen Washing kit (NimbleGen Systems; Roche) protocol. The array image was acquired using an Axon GenePix 4000B (Axon Instruments, Inc., Union City, CA, USA) laser scanner at a 5- $\mu \mathrm{m}$ resolution and the intensity data were extracted using the NimbleScan software (NimbleGen Systems; Roche). The data was further examined using NexuExp software (BioDiscovery, El Segundo, CA, USA). Gene expression changes that were greater or less than two-fold as compared with the control group, and with a $\mathrm{P}<0.01$, were considered to indicate a statistically significant difference in the expressed genes between the samples.

Quantitative polymerase chain reaction ( $q P C R) . \mathrm{SYBR}^{\circledR}$ Green qPCR primers were designed using Beacon Designer software version 2 (PREMIER Biosoft International, Palo Alto, CA, USA) with the following sequences: forward, 5'-AGTCGTGGG AAGAGGGAACT-3', and reverse, 5'-CCCTGGAAGTTGTTC ATGCT-3' for adrenomedullin (Adm); forward, 5'-ACAGAG CATGACCCTGAACC-3', and reverse, 5'-CCGTTGCTGGAC TGGATTAT-3' for Jun; forward, 5'-CAAGACAAAAGCGTG GTTGA-3', and reverse, 5'-TCTTCCTGAGTCCCTCCTGA-3' for Junb; forward, 5'-AATGGAGGTGATGGCAGACA-3', and reverse, 5'-GAGCAACCCACAGAGTACCT-3' for c-FBJ osteosarcoma ( $c$-Fos); forward, 5'-GGGTCACTGGTGTTT GAGGA-3', and reverse, 5'-CCTCGGCTTTTGTGATGGAC-3' for activating transcription factor 3 (Atf3) and forward, 5'-CTC AGCCAATTGTCCCAACC-3', and reverse, 5'-AGGTAAGCA AGGCAGATGGT-3' for dual specificity phosphatase 1 (Duspl) genes, . SYBR Green reactions were performed using the SYBR Green Supermix (BioRad, Hercules, CA, USA). The qPCR reactions were then performed using the BioRad iCycler iQ Real-Time Detection system (BioRad). The cycling conditions were as follows; $3 \mathrm{~min}$ at $95^{\circ} \mathrm{C}, 15 \mathrm{sec}$ at $95^{\circ} \mathrm{C}$ and $45 \mathrm{sec}$ at $55^{\circ} \mathrm{C}$ for 45 cycles. The relative expression levels of Adm, Jun, Junb, c-fos, Atf3 and Duspl were analyzed using the iCycle iQ system software and presented as a ratio to the expression of the housekeeping gene, tubulin. Each sample was replicated twice from three independent sets of RNA preparations.

Statistical analysis. All values are expressed the mean + standard deviation. The results of the gene expression levels across

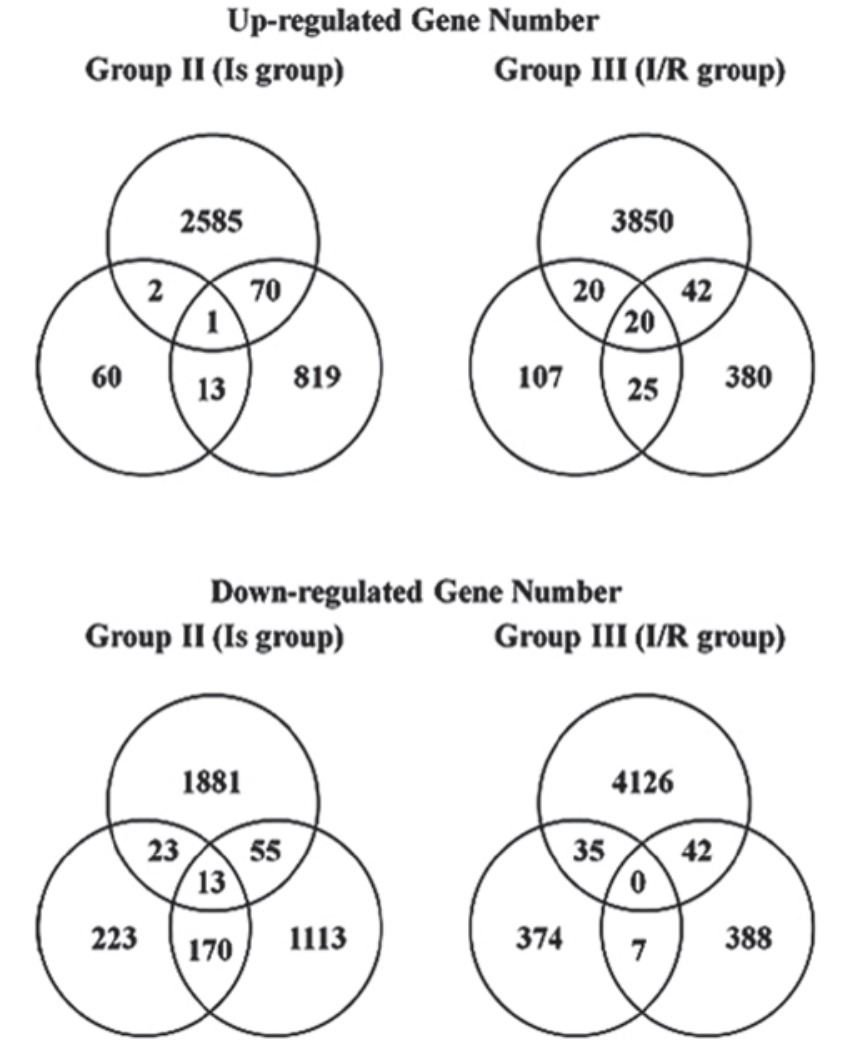

Figure 1. Venn diagram of the probe sets differentially expressed in the kidney, intestine, and skeletal muscle rat models. Is, ischemia group (group II); I/R, ischemia / reperfusion group (group III).

the different groups were analyzed by analysis of variance with post-hoc comparison using Kruskal-Wallis test. A $\mathrm{P}<0.05$ was considered to indicate a statistically significant difference. The statistical analysis was performed using SPSS 17.0 (SPSS Inc., Chicago, IL, USA).

\section{Results}

Gene expression profiling in $I / R$ models. The microarray compared the expression profile of $>21486$ genes, using the Nexus Expression $^{\mathrm{TM}}$ analysis software (BioDiscovery). Each organ had a different number of genes that were differentially expressed during the I/R condition (Table I). As compared with the sham operation group, in the intestinal model, there were 76 genes upregulated and 429 genes downregulated in the ischemia-only group (group II) and 172 genes upregulated and 416 genes downregulated in the I/R group (group III). In 
Table II. Common up- and downregulated genes in each group.

A, Commonly upregulated genes in the Is group

\begin{tabular}{|c|c|c|c|c|c|c|}
\hline Probes & Name & Gene symbol & Chromosome & $\begin{array}{l}\text { Intestine } \\
\text { log ratio }\end{array}$ & $\begin{array}{l}\text { Kidney } \\
\text { log ratio }\end{array}$ & $\begin{array}{l}\text { Muscle } \\
\text { log ratio }\end{array}$ \\
\hline \multicolumn{7}{|l|}{ Transcription factor } \\
\hline NM_001024781 & $\begin{array}{l}\text { SRY-box containing } \\
\text { gene } 18\end{array}$ & Sox 18 & 3 & 1.2144 & 1.5284 & 1.0687 \\
\hline
\end{tabular}

$\mathrm{B}$, Commonly downregulated genes in the Is group

\begin{tabular}{|c|c|c|c|c|c|c|}
\hline Probes & Name & Gene symbol & Chromosome & $\begin{array}{l}\text { Intestine } \\
\text { log ratio }\end{array}$ & $\begin{array}{l}\text { Kidney } \\
\log \text { ratio }^{\text {a }}\end{array}$ & $\begin{array}{l}\text { Muscle } \\
\log \text { ratio }^{a}\end{array}$ \\
\hline \multicolumn{7}{|l|}{ Apoptosis } \\
\hline AF517560 & Caspase 9 & Casp9 & 5 & -1.0343 & -1.1199 & -1.1743 \\
\hline \multicolumn{7}{|l|}{ Signaling pathway } \\
\hline NM_144730 & GATA binding protein 4 & Gata4 & 15 & -1.2419 & -1.1162 & -1.0620 \\
\hline NM_024400 & $\begin{array}{l}\text { A disintegrin-like and } \\
\text { metallopeptidase with } \\
\text { thrombospondin type } 1 \\
\text { motif, } 1\end{array}$ & Adamts1 & 11 & -1.0472 & -1.1230 & -1.7934 \\
\hline NM_001000131 & Olfactory receptor 50 & Olr50 & 1 & -1.4110 & -2.0005 & -1.1353 \\
\hline \multicolumn{7}{|l|}{ Adhesion molecules } \\
\hline NM_012702 & $\begin{array}{l}\text { Carcinoembryonic } \\
\text { antigen-related cell } \\
\text { adhesion molecule } 3\end{array}$ & Ceacam3 & 1 & -1.1057 & -1.8840 & -1.0048 \\
\hline \multicolumn{7}{|l|}{ Protein coding } \\
\hline NM_001037518 & Defensin beta 23 & Defb23 & 3 & -1.0558 & -1.4811 & -1.0821 \\
\hline XM_575765 & $\begin{array}{l}\text { Similar to suppressor } \\
\text { of initiator codon } \\
\text { mutations, related } \\
\text { sequence } 1\end{array}$ & RGD1560994 & 5 & -1.0367 & -1.3214 & -1.2535 \\
\hline XM_001053867 & $\begin{array}{l}\text { Hypothetical protein } \\
\text { LOC679650 }\end{array}$ & LOC679650 & 4 & -1.2825 & -1.3383 & -1.7832 \\
\hline XM_001058313 & $\begin{array}{l}\text { Hypothetical protein } \\
\text { LOC680675 }\end{array}$ & LOC680675 & 2 & -1.1665 & -1.8205 & -1.3372 \\
\hline XM_001066721 & $\begin{array}{l}\text { Hypothetical protein } \\
\text { LOC688387 }\end{array}$ & LOC688387 & 15 & -1.3056 & -1.5512 & -2.0187 \\
\hline XM_001071268 & $\begin{array}{l}\text { Hypothetical protein } \\
\text { LOC689585 }\end{array}$ & LOC689585 & 14 & -1.2310 & -1.2955 & -3.3694 \\
\hline XM_001075138 & $\begin{array}{l}\text { Hypothetical protein } \\
\text { LOC690663 }\end{array}$ & LOC690663 & 7 & -1.0482 & -1.3571 & -1.8585 \\
\hline XM_001079793 & $\begin{array}{l}\text { Hypothetical protein } \\
\text { LOC691833 }\end{array}$ & LOC691833 & 7 & -1.2318 & -2.5800 & -1.1789 \\
\hline
\end{tabular}

$\mathrm{C}$, Commonly upregulated genes in the I/R group

\begin{tabular}{|c|c|c|c|c|c|c|}
\hline Probes & Name & Gene symbol & Chromosome & $\begin{array}{l}\text { Intestine } \\
\log \text { ratio }^{\mathrm{a}}\end{array}$ & $\begin{array}{c}\text { Kidney } \\
\text { log ratio }\end{array}$ & $\begin{array}{c}\text { Muscle } \\
\text { log ratio }\end{array}$ \\
\hline
\end{tabular}

Toll-like receptor signaling pathway

MAPK pathway

BC078738

Jun oncogene

Jun

5

1.5935

2.2445

4.1868 
Table II. Continued.

\begin{tabular}{|c|c|c|c|c|c|c|}
\hline Probes & Name & Gene symbol & Chromosome & $\begin{array}{l}\text { Intestine } \\
\log \text { ratio }^{a}\end{array}$ & $\begin{array}{l}\text { Kidney } \\
\log \text { ratio }^{a}\end{array}$ & $\begin{array}{l}\text { Muscle } \\
\log \text { ratio }^{\mathrm{a}}\end{array}$ \\
\hline ВС078903 & $\begin{array}{l}\text { Activating transcription } \\
\text { factor } 3\end{array}$ & Atf3 & 13 & 2.8074 & 3.3245 & 4.4505 \\
\hline NM_012715 & Adrenomedullin & $A d m$ & 1 & 1.0172 & 1.5631 & 1.6412 \\
\hline NM_021836 & Jun-B oncogene & Junb & 19 & 1.8383 & 1.2752 & 3.8498 \\
\hline NM_022197 & $\begin{array}{l}\text { FBJ osteosarcoma } \\
\text { oncogene }\end{array}$ & Fos & 6 & 2.1100 & 3.9940 & 2.1579 \\
\hline NM_053769 & $\begin{array}{l}\text { Dual specificity } \\
\text { phosphatase } 1\end{array}$ & Duspl & 10 & 1.9596 & 1.1450 & 2.7656 \\
\hline \multicolumn{7}{|l|}{$\mathrm{NF}-\kappa \mathrm{B}$ pathway } \\
\hline XM_221537 & Nfkbiz & Nfkbiz & 11 & 1.3469 & 1.8728 & 3.9178 \\
\hline NM_017259 & B-cell translocation gene 2 & Btg2 & 13 & 1.1585 & 1.1407 & 1.6365 \\
\hline NM_022542 & $\begin{array}{l}\text { Ras homolog gene family, } \\
\text { member B }\end{array}$ & Rhob & 6 & 1.9195 & 1.2385 & 2.9469 \\
\hline L25925 & Cyclooxygenase-2 & $\operatorname{Cox} 2$ & 13 & 2.7905 & 2.1123 & 1.0614 \\
\hline \multicolumn{7}{|l|}{$\begin{array}{l}\text { Cell proliferation } \\
\text { and differentiation }\end{array}$} \\
\hline ВC070878 & $\begin{array}{l}\text { Polo-like kinase } 2 \\
\text { (Drosophila) }\end{array}$ & $P l k 2$ & 2 & 1.6628 & 1.8470 & 1.7820 \\
\hline NM_031642 & Kruppel-like factor 6 & Klf6 & 17 & 1.1219 & 1.6330 & 1.6238 \\
\hline NM_024388 & $\begin{array}{l}\text { Nuclear receptor } \\
\text { subfamily } 4\end{array}$ & $N r 4 a l$ & 7 & 2.2865 & 1.6597 & 4.9402 \\
\hline \multicolumn{7}{|l|}{ Protein binding } \\
\hline NM_001003401 & $\begin{array}{l}\text { Ectodermal-neural } \\
\text { cortex } 1\end{array}$ & Encl & 2 & 1.7737 & 1.2063 & 3.1094 \\
\hline NM_001009541 & $\begin{array}{l}\text { Immediate early } \\
\text { response } 2\end{array}$ & Ier 2 & 19 & 1.6128 & 2.0729 & 1.8945 \\
\hline \multicolumn{7}{|l|}{ Cytokine } \\
\hline NM_031512 & Interleukin 1 beta & $I L-1 \beta$ & 3 & 1.0116 & 1.0882 & 2.1743 \\
\hline NM_053565 & $\begin{array}{l}\text { Suppressor of cytokine } \\
\text { signaling } 3\end{array}$ & Socs3 & 10 & 1.3094 & 1.2761 & 1.4714 \\
\hline NM_012945 & $\begin{array}{l}\text { Heparin-binding EGF-like } \\
\text { growth factor }\end{array}$ & Hbegf & 18 & 2.8253 & 1.5989 & 2.3193 \\
\hline \multicolumn{7}{|l|}{$\begin{array}{l}\text { Circulation and } \\
\text { coagulation }\end{array}$} \\
\hline NM_173141 & $\begin{array}{l}\text { Tissue factor pathway } \\
\text { inhibitor } 2\end{array}$ & Tfpi2 & 4 & 1.0111 & 1.3111 & 2.7850 \\
\hline NM_001003403 & $\begin{array}{l}\text { Vascular early response } \\
\text { gene protein }\end{array}$ & Verge & 4 & 1.0461 & 1.1412 & 3.5718 \\
\hline
\end{tabular}

${ }^{\mathrm{a}} \log _{2}$ ratio. Is, ischemia group (group II); I/R, ischemia/reperfusion group (group III).

the renal model, there were 903 genes upregulated and 1351 genes downregulated in the ischemia only group and 467 genes upregulated and 437 genes downregulated in the I/R group. In the skeletal muscle model, there were 2658 genes upregulated and 1972 genes downregulated in the ischemia only group and 3932 genes upregulated and 4203 genes down-regulated in I/R group (Table I).

Comparisons of the gene expression profiling in different organ models. The details of the up- and downregulated genes were 

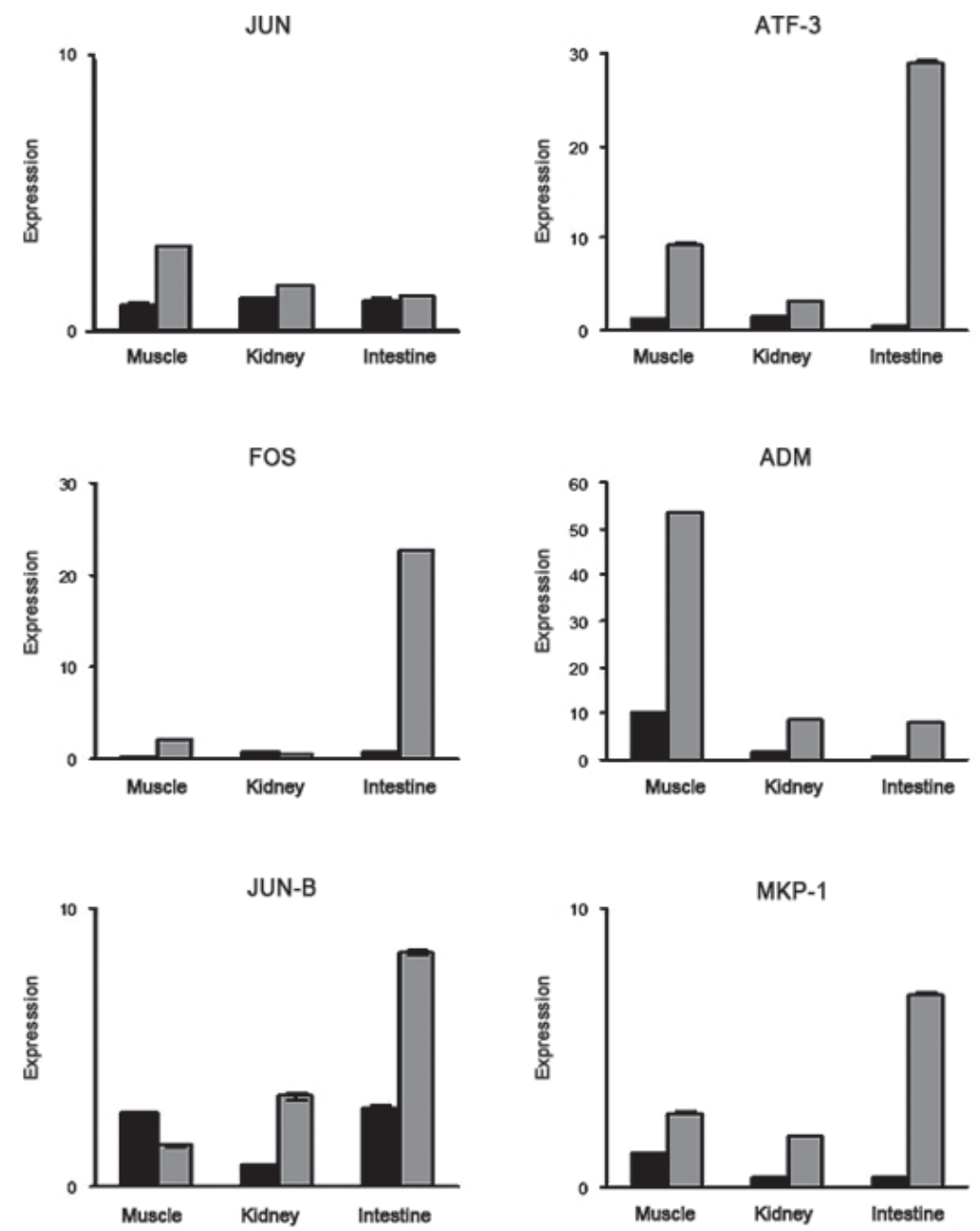

Figure 2. Selected gene expression status in skeletal muscle, kidney and intestine, was examined by quantiative polymerase chain reaction (qPCR). The black bars represent the expression of the ischemia-only group (II) and the gray bars represent the expression of the I/R group (III). The expression levels of each gene in each organ detected by qPCR and microarray experiments were comparable. ATF-3, activating transcription factor 3; FOS, FBJ osteosarcoma; ADM, adrenomedullin; $M K P$-1, mitogen-activated protein kinase phosphatase-1.

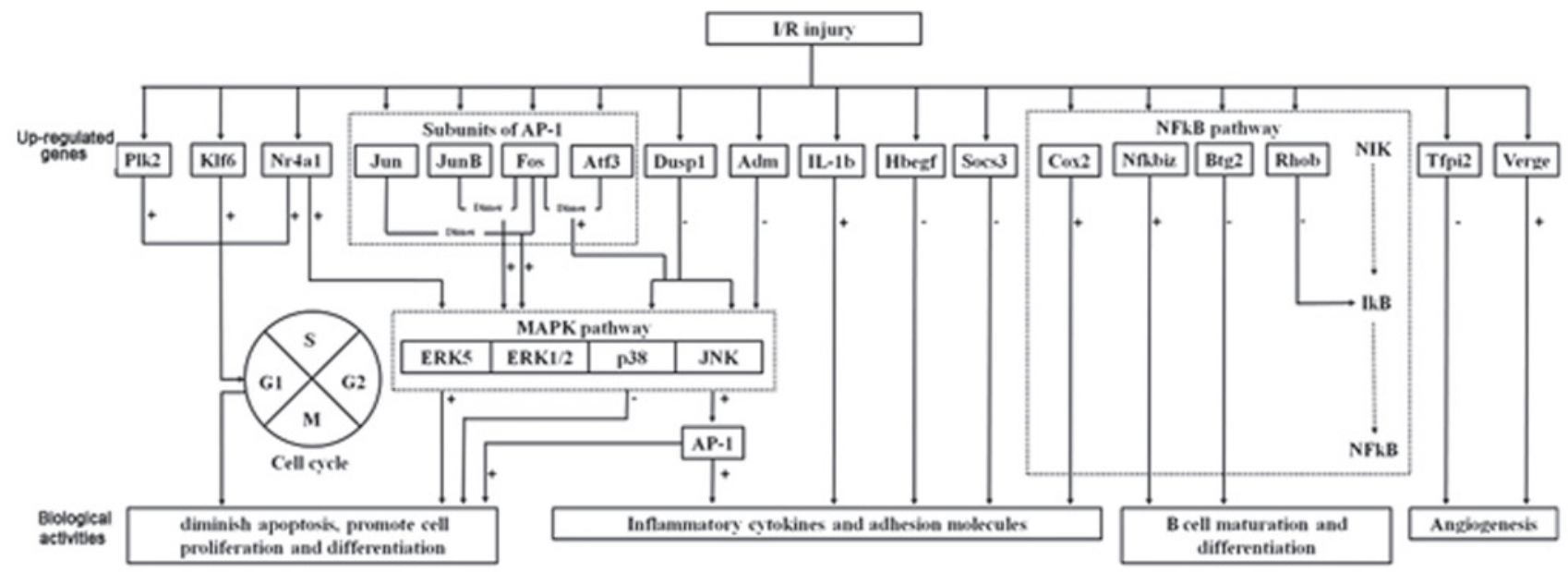

Figure 3. Schematic diagram illustrating the proposed complex mechanisms of I/R conditions. For MAPK pathway modulation, the selective inhibition of the p38 and JNK pathway can be achieved by enhanced expression of Dusp1 and Adm, together with inhibition of AP-1. For NFkB pathway modulation, enhanced expression of Btg2 and Rhob together with inhibition of Nfkbiz and the downstream target gene Cox 2 can diminish the inflammatory response. The action towards reducing apoptosis and promotion of cellular proliferation can be achieved through the upregulation of Klf6 and Plk2, and inhibition of the p38 pathway. Control of cytokine and adhesion molecules may be achieved through direct inhibition of $I L-1 \beta$ and enhanced expression of $H b e g f$ and Socs 3 . For modulation of microcirculation, the upregulation of Verge and downregulation of Tfpi2 may promote angiogenesis. I/R, ischemia/reperfusion; ATF-3,

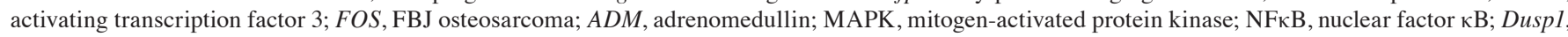
dual specificity phosphatase 1; AP-1, activator protein 1; Btg2, B-cell translocation gene 2; Rhob, Ras homolog gene family member B; Cox2, cyclooxygenase 2; Klf6, Kruppel-like factor 6; Plk2, polo-like kinase 2; IL, interleukin; Hbegf, heparin-binding EGF-like growth factor; Socs3, suppressor of cytokine signaling 3; Verge, vascular early response gene protein; Tfpi2, tissue factor pathway inhibitor 2. 
markedly different between the organs. Fig. 1 shows the Venn diagram of the genes that were differentially up- and downregulated in the Is and I/R groups in all three models. As for the common up- or downregulated genes, one and 13 gene probe sets were up- and downregulated in the Is group, respectively; 20 gene probe sets were upregulated in the $\mathrm{I} / \mathrm{R}$ group and no genes were downregulated in the $\mathrm{I} / \mathrm{R}$ group. The details of the common up and downregulated genes are shown in Table II.

Validation of target gene expression in the I/R injury model using $q P C R$. In order to confirm the validity of the microarray findings with regard to the genes up- or downregulated in common in all three organ models, the same RNA samples of the three organs used for the microarrays were subjected to qPCR. Primers were selected for six representative genes associated with the MAPK pathway, including Atf3 (GenBank: NM_012912, BC078903), Jun (GenBank: BC078738), Jun b (GenBank: NM_021836), c-Fos (GenBank: NM_022197), Duspl (GenBank: NM_053769) and Adm (GenBank: NM_012715). The results of the qPCR expression are shown in Fig. 2. The majority of the qPCR results confirmed the upregulated gene expression. Selected gene expression status in three different organs was additionally examined by qPCR. The expression levels of each gene in each organ detected by qPCR and microarray experiments were comparable.

\section{Discussion}

The detailed mechanisms of I/R injury in individual organs have not been fully elucidated due to the molecular complexity of the condition. The present study used a single organ model and gene expression profiling method to identify specific molecules that may be important in I/R injury at an early ischemia and reperfusion time-point. After $1 \mathrm{~h}$ ischemia, there was only one commonly upregulated gene (Sox18; NM_001024781) and 13 downregulated genes. Overexpression of Sox18 in blood vascular endothelial cells was previously reported to induce angiogenesis and lymphangiogenesis, which is associated with the ischemic response of organs. Sox 18 therefore has the potential be an organ-ischemic marker (13). Of the 13 common downregulated genes, Gata4 (NM_144730) is a downstream gene of the MAPK pathway and its downregulation may represent the inactive status of the extracellular signal-regulated protein kinase (ERK) 1/2 pathway, which corresponded to the inactivity of nuclear factor kappa-light-chain-enhancer of activated B-cells (NFkB) and activator protein (AP)-1 at this time-point (14). Ischemic insult also induces apoptosis and angiogenesis in order to respond to the hypoxic status, thus the downregulation of the adversely effected genes, including Casp9 (AF517560), Adamtsl (NM_024400) and Ceacam3 (NM_012702) are expected (15-17).

After $1 \mathrm{~h}$ reperfusion, additional biological activities were present, in which the interacting functions increased the biological complexity. There were 20 commonly upregulated gene probe sets in the I/R group. The majority of genes were not significantly upregulated during the initial $1 \mathrm{~h}$ of ischemia. Among these genes, several were involved in the MAPK and $\mathrm{NF}$ B B pathways. These two pathways may serve as the common pathways between the three organs at this time-point and modulate the biochemical response towards I/R injury $(18,19)$.
Six genes were identified that were involved in the MAPK signaling pathway. Four of these were associated with the heterodimeric protein AP-1, Jun, Atf3, Jun b, and Fos. AP-1 is one of the end targets of the MAPK signaling pathway, and is considered to mediate I/R-induced gene expression since numerous subunit genes are known to mediate either proliferation, differentiation, or apoptosis (Jun family predominant) by altering the expression levels of cytokines, neurotransmitters, and other intercellular signaling molecules $(20,21)$. AP-1 is additionally known to function in the process of T-cell activation, which is a key process in transplant immunology (22). In addition, AP-1 activates numerous downstream genes which are implicated in organ damage $(23,24)$. AP-1 consists of three major subfamilies, including Jun, Fos, and Atf (25). In the early phase following $\mathrm{I} / \mathrm{R}$ stress, the high expression levels of Jun and Atf activate the JNK and P38 pathways, promoting apoptosis. The high expression levels of Jun and Fos activate the ERK1/2 pathway to promote cellular proliferation (26). The data from the present study showed that there was a higher expression of Jun, Junb and Fos, but no significant difference in the expression of Atf 3 . This expression pattern was compatible with the previously described theories of apoptosis (27). Atf3, however, was found to be a common gene with higher expression (26). Atf3 is a stress-inducible gene that encodes a member of the ATF/cyclic adenosine monophosphate response element binding protein family of transcription factors (28). Atf 3 mRNA was observed to increase in expression within $2 \mathrm{~h}$ following exposure of cells to stress signals, and therefore, $A f t 3$ is a suitable candidate for further analysis in I/R injury.

The MAPK pathway may additionally be mediated during I/R injury by higher expression levels of Dusp1 and Adm, which downregulate the MAPK pathway. Duspl is an oxidative stress-inducible gene that acts as a negative regulator of the JNK and p38 pathways (29). Adm selectively inhibits the JNK pathway, therefore the two genes may act in opposition to AP-1 (30). The adjustment of their expression may facilitate a reduction in I/R injury.

The NFKB pathway is another important pathway that responds to $I / R$ injury at this time-point. Ischemic insult activates $\mathrm{NF} \kappa \mathrm{B}$-inducing kinase, which degrades IкB kinase

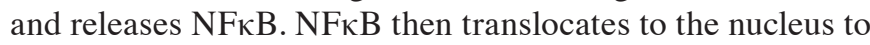
induce bioactivities including promotion of transcription and activation of adhesion molecules, cytokines and maturing of $\mathrm{B}$ cells (31). According to the presented database, the upregulation of Rhob (NM_022542) may repress NFkB signaling by inhibiting dissociation and subsequent degradation of I $\mathrm{B}$, therefore further diminishing the downstream inflammatory response. Two genes were additionally identified to modulate B-cells. Btg2 (NM_017259), the p53-transcriptional target, is an anti-proliferative B-cell translocation gene. Over-expression of $\mathrm{Btg} 2$ has a protective role, inducing B-cell depletion, which can further reduce the inflammatory response. Conversely, Nfkbiz (GenBank: XM_221537) activates B-cell proliferation and differentiation to enhance the inflammatory response (32). The present study additionally identified prostaglandin-endoperoxide synthase 2 (Cox2; GenBank: L25925, NM_017232) to be upregulated in the three organ models. Cox2 is an enzyme that catalyzes the initial step of the synthesis of inflammatory prostaglandins from arachidonic acid. The upregulation of Cox2 can activate 
the $\mathrm{NF \kappa B}$ pathway and perform additional downstream bioactivities (33).

The cytokines and adhesion molecules triggered by different signaling pathways function to initiate the inflammatory response towards $\mathrm{I} / \mathrm{R}$ insult. According to the presented database, only interleukin $1 \beta$ (IL-1 $\beta$; NM_031512) was identified to be upregulated in all three organ models. However, Hbegf (GenBank: NM_012945) and Socs3 (GenBank: NM_053565) were two genes identified that act as a negative controller, eliciting protective effects against cytokine and adhesion molecules, and diminishing the inflammatory response.

Other genes were identified in the present study that have not been previously associated with I/R injury, however may be functional in the I/R response. These genes included Verge (GenBank:NM_001003403) and Tfpi2 (GenBank:NM_173141), which were noted to be associated with angiogenesis and capillary endothelial and microcirculation dysfunction, as well as Plk2 (GenBank: NM_031821, BC070878), Klf6 (NM_031642) and Nr4al (NM_024388), which are involved in the G1 phase of the cell cycle and can promote cellular proliferation and prevent apoptosis (34). The schematic diagram in Fig. 3 illustrates the proposed complex mechanisms of I/R conditions.

In the present study, the uniquely affected genes in the three organ models in both ischemia and reperfusion status were identified and compared. Among these genes, several were identified to be associated with the MAPK and NFкB signaling pathways. The present study focused on only two time-points following I/R insult; therefore, the kinetic changes of the specific genes require further investigation. This study provided fundamental information to the understanding of the key biomechanical changes during I/R injury.

\section{Acknowledgements}

The authors would like to thank the Chang Gung Memorial Hospital for financial support (nos. CMRPG470041, CMRPG4B0021, CMRPG4A0101 and CMRPG4A0102), and the Chang Gung Memorial Hospital Urology Laboratory.

\section{References}

1. Ames A: Cerebral ischemia. II. The no-reflow phenomenon. Am J Pathol 52: 437, 1968.

2. Hsieh Y-H, Huang S-S, Wei F-C and Hung L-M: Resveratrol attenuates ischemia - reperfusion-induced leukocyte - endothelial cell adhesive interactions and prolongs allograft survival across the MHC barrier. Circ J 71: 423-428, 2007

3. Wei W, Wei FC and Hung L-M: Diazoxide ameliorates microcirculatory disturbances through PKC-dependent pathway in I/R-injured rat cremaster muscles. J Biomed Sci 12: 521-529, 2005.

4. Kobrin SM: Diabetic nephropathy. Dis Mon 44: 214-234, 1998.

5. Shihab FS: Cyclosporine nephropathy: pathophysiology and clinical impact. Semin Nephrol 16: 536-547, 1996.

6. Wu X, Pang ST, Sahlin L, et al: Gene expression profiling of the effects of castration and estrogen treatment in the rat uterus. Biol Reprod 69: 1308-1317, 2003.

7. Pang ST, Dillner K, Wu X, et al: Gene expression profiling of androgen deficiency predicts a pathway of prostate apoptosis that involves genes related to oxidative stress. Endocrinology 143 4897-4906, 2002.

8. Wu MS, Yang CW, Chang CT, Bens M and Vandewalle A: Cyclosporin increases the density of angiotensin II subtype 1 (AT1) receptors in mouse medullary thick ascending limb cells. Nephrol Dial Transplant 18: 1458-1465, 2003.
9. Wu MS, Bens M, Yu HM and Vandewalle A: Cyclosporine reduces basolateral, but not apical, nitric oxide secretion in medullary thick ascending limb cells. Transpl Int 13: S321-S323 2000.

10. Mason J: The pathophysiology of Sandimmune (cyclosporine) in man and animals. Pediatr Nephrol 4: 554-574, 1990.

11. Luo CC, Chen HM, Chiu CH, Lin JN and Chen JC: Effect of $\mathrm{N}(\mathrm{G})$-nitro-L-arginine methyl ester on intestinal permeability following intestinal ischemia-reperfusion injury in a rat model. Biol Neonate 80: 60-63, 2001.

12. Basile DP, Fredrich K, Alausa M, et al: Identification of persistently altered gene expression in the kidney after functional recovery from ischemic acute renal failure. Am J Physiol Renal Physiol 288: F953-F963, 2005.

13. François M,Caprini A, Hosking B, et al: Sox 18 induces development of the lymphatic vasculature in mice. Nature 456: 643-647, 2008.

14. Liang Q, Wiese RJ, Bueno OF, et al: The transcription factor GATA4 is activated by extracellular signal-regulated kinase 1-and 2-mediated phosphorylation of serine 105 in cardiomyocytes. Mol Cell Biol 21: 7460-7469, 2001.

15. Park M-T, Choi J-A, Kim M-J, et al: Suppression of extracellular signal-related kinase and activation of p38 MAPK are two critical events leading to caspase-8- and mitochondria-mediated cell death in phytosphingosine-treated human cancer cells. J Biol Chem 278: 50624-50634, 2003.

16. Basile DP, Fredrich K, Chelladurai B, Leonard EC and Parrish AR: Renal ischemia reperfusion inhibits VEGF expression and induces ADAMTS-1, a novel VEGF inhibitor. Am J Physiol Renal Physiol 294: F928-F936, 2008.

17. Skubitz KM and Skubitz A: Interdependency of CEACAM-1, $-3,-6$, and -8 induced human neutrophil adhesion to endothelial cells. J Transl Med 6: 78, 2008.

18. Qi M and Elion EA: MAP kinase pathways. J Cell Sci 118: 3569-3572, 2005.

19. Mullonkal CJ and Toledo-Pereyra LH: Akt in ischemia and reperfusion. J Invest Surg 20: 195-203, 2007.

20. Karin M: The regulation of AP-1 activity by mitogen-activated protein kinases. J Biol Chem 270: 16483-16486, 1995.

21. Shaulian E and Karin M: AP-1 as a regulator of cell life and death. Nat Cell Biol 4: E131-136, 2002.

22. Halloran PF: Immunosuppressive drugs for kidney transplantation. N Engl J Med 351: 2715-2729, 2004.

23. Yeh KY, Yeh M, Glass J and Granger DN: Rapid activation of NF-kappaB and AP-1 and target gene expression in postischemic rat intestine. Gastroenterology 118: 525-534, 2000.

24. Karin M: The regulation of AP-1 activity by mitogen-activated protein kinases. J Biol Chem 270: 16483-16486, 1995.

25. Shima Y, Tajiri T, Taguchi T and Suita S: Increased expression of c-fos and c-jun in the rat small intestinal epithelium after ischemia-reperfusion injury: a possible correlation with the proliferation or apoptosis of intestinal epithelial cells. J Pediatr Surg 41: 830-836, 2006

26. Hai T and Hartman MG: The molecular biology and nomenclature of the activating transcription factor/cAMP responsive element binding family of transcription factors: activating transcription factor proteins and homeostasis. Gene 273: 1-11, 2001.

27. Ameyar M, Wisniewska M and Weitzman JB: A role for AP-1 in apoptosis: the case for and against. Biochimie 85: 747-752, 2003.

28. Kang Y, Chen C-R and Massagué J: A self-enabling TGFbeta response coupled to stress signaling: Smad engages stress response factor ATF3 for Id1 repression in epithelial cells. Mol Cell 11: 915-926, 2003.

29. Weng Y, Shen F, Li J, Shen Y and Zhang X: Expression changes of mitogen-activated protein kinase phosphatase-1 (MKP-1) in myocardium of streptozotocin-induced diabetic rats. Exp Clin Endocrinol Diabetes 115: 455-460, 2007.

30. Yoshimoto T, Fukai N, Sato R, et al: Antioxidant effect of adrenomedullin on angiotensin II-induced reactive oxygen species generation in vascular smooth muscle cells. Endocrinology 145: 3331-3337, 2004.

31. Chen J, Crispín JC, Tedder TF, Dalle Lucca J and Tsokos GC: B cells contribute to ischemia/reperfusion-mediated tissue injury. J Autoimmun 32: 195-200, 2009.

32. Liu L, Sakai T, Sano N and Fukui K: Nucling mediates apoptosis by inhibiting expression of galectin-3 through interference with nuclear factor kappaB signalling. Biochem J 380: 31-41, 2004.

33. Malek HA and Saleh DM: Cyclooxygenase-2 inhibitor celecoxib in a rat model of hindlimb ischemia reperfusion. Can J Physiol Pharmacol 87: 353-359, 2009.

34. Hollander MC, Poola-Kella S and Fornace AJ: Gadd34 functional domains involved in growth suppression and apoptosis. Oncogene 22: 3827-3832, 2003. 